\title{
Perfomatividad en identidad de género y violencia doméstica: el caso de las mujeres del Centro de la Mujer de Talcahuano
}

\begin{abstract}
Alina Muñoz ${ }^{1}$
Fecha de recepción: 20 de noviembre de 2014

Fecha de aprobación: 18 de diciembre de 2014

\section{Resumen}

La categoría "género" en mujeres que han vivenciado situaciones de violencia doméstica, se transforma mediante un discurso que desde la individualidad se comparte, generando posibilidades de rupturas y de cambio. En el caso de las mujeres del Centro de la Mujer de Talcahuano, tenemos un grupo de sujetas que se conocen como beneficiarias de un programa de violencia doméstica, cuyo objetivo es la búsqueda de la reducción de la violencia contra la mujer, especialmente aquella que se produce en el contexto de relaciones de pareja, mediante la implementación de un modelo psicosocial que realiza prevención mediante trabajo comunitario y que atiende a mujeres mayores

En este trabajo, y mediante un estudio cualitativo generado a partir de la construcción de producciones narrativas, se da cuenta de cómo el género se subjetiviza y se construye como un elemento de doble apropiación, en el que coexisten elementos que dan continuidad a través de la reiteración y la nominación al relato tradicional del género (maternidad), en conjunto con aquellos que lo agencian y potencian como un eje articulador del cambio, tanto en el autoconcepto como en lo que a relaciones de género se refiere.
\end{abstract} de 18 años víctimas de violencia doméstica.

Palabras clave: identidad de género, producciones narrativas, subjetivación, performatividad.

\section{Performativity in gender identity and domestic violence: the case of Women in the Center for women in Talcahuano}

\begin{abstract}
The gender category in women who have experienced domestic violence situations is transformed by a speech shared from individuality, generating
\end{abstract}

Socióloga, Magíster en Psicología Comunitaria, Magíster (c) Investigación Social y Desarrollo. Académica, Universidad San Sebastián, Concepción, Chile. Alinamunoz.sociologa@gmail.com 
possibilities of ruptures and change. In the case of women from the Center for Women, we have a group of subjects known as beneficiaries of a domestic violence program which aims to reduce violence against women, especially that which occurs in the context of relationships, by implementing a psychosocial model that promotes prevention through community service and serves women over the 18 years old who are victims of domestic violence. By a qualitative work generated from the construction of narrative productions, this paper shows how gender is subjectivised and constructed as a double appropriation element with coexistingelements that give continuity through repetition and nomination of gender traditional story (maternity), together with those which promote it and strengthen it as a driver of change, as self-concept and in gender relations.

Keywords: Gender identity, narrative productions, subjectivation, performativity.

\title{
Performatividade na identidade de gênero e violência doméstica: o caso das Mulheres do Centro de Talcahuano
}

\begin{abstract}
Resumo
A categoria gênero em mulheres que tem sofrido situações de violência doméstica transformou-se mediante um discurso que desde a individualidade se compartilha gerando possibilidades de rupturas e mudanças. No caso das mulheres do Centro da Mulher, temos um grupo de sujeitos conhecidos como beneficiárias de um programa de violência doméstica que tem como objetivo procurar a redução da violência contra as mulheres, especialmente a que acontece no contexto das relações entre casais, através da implementação de um modelo psicossocial que realiza a prevenção através do serviço comunitário e atende a mulheres com mais de 18 anos, vítimas de violência doméstica.

Neste trabalho, através de um trabalho qualitativo gerado a partir da construção de produções narrativas, se percebe como o gênero se subjetiva e é construída como um elemento de dupla apropriaçãoonde coexistem elementos que dão continuidade através da reiteração e nomeação ao relato tradicional do gênero (maternidade), juntamente com aqueles que o agenciam e potenciam como um eixo articulador do cambio, tanto no autoconceito, tanto quanto as relações de gênero referem-se.
\end{abstract}

Palavras-chave: identidade de gênero, produções narrativas, subjetivação, performatividade.

\section{Introducción}

El presente escrito tiene por objetivo presentar algunas reflexiones teóricas en torno a la construcción de identidad de género, a partir de los resultados obtenidos en la investigación “Construcción de narrativas de identidad de género en mujeres 
víctimas de violencia doméstica: el caso de las mujeres del Centro de la Mujer de Talcahuano". Ello con la finalidad de generar una narrativa de identidad de género que permita comprender los modos, formas, ejes, discursos y actos performativos que dan vida a la emergencia, configuración y transformación de las mujeres que han vivido situaciones de violencia y han sido intervenidas psicosocialmente de manera colectiva.

Nociones que permitieron, además, abordar diversas posiciones respecto de la violencia sexo-amorosa y desde ahí construir una mirada sobre las identidades de género que no reproduce la teorización, sino que, más bien, "surge de condiciones semióticomateriales concretas de actores sociales en un contexto [social]" (Martínez, Guzmán y Montenegro, 2009, p. 7), que en este caso fue y es la comunidad Centro de la Mujer de la comuna de Talcahuano.

\section{Aspectos metodológicos}

La investigación cualitativa y sus técnicas de producción de datos de investigación (producciones narrativas) no deben adoptarse como un "recetario" que baja desde epistemologías hipotético-deductivas. La investigación cualitativa y las producciones narrativas surgen desde la epistemología socioconstruccionista, que asume a la realidad como una construcción social constante, en la cual las metodologías utilizadas deben ser abordadas desde un proceso de co-construcción, en el marco de la búsqueda de una "verdad participativa" (Gadamer, 1975). Allí la construcción del conocimiento investigativo se produce mediante un diálogo reflexivo y permanente entre "informantes e investigadores". Es un conocimiento que se construye en y desde la vida y la experiencia de las personas, es la producción de un conocimiento situado, esto desde la ontología de que no hay una realidad objetiva, de que esta se crea socialmente, que su producción es específica para cada contexto y que, en este sentido, el conocimiento investigativo surge desde la interacción social entre sujetos sociales -"investigador e 
investigados"-. Por lo demás, esta será específica para cada contexto, dependiendo de su composición sociocultural (Haraway, 1991; Martínez, Guzmán y Montenegro, 2009).

Las producciones narrativas surgen desde la perspectiva de los "conocimientos situados", propuestos por Donna Haraway (1991), caracterizados como una herramienta metodológica que permite interpelar a las mujeres hacia un ejercicio de reflexividad en torno a la construcción de su identidad, pero incluso desde aspectos desconocidos por las propias mujeres, para construir un relato colectivo generado a partir de elementos intra e intersubjetivos y que, considerando las particularidades, permite comprender los elementos que tensionan y transforman sus identidades (Marinas, 2007).

Es importante mencionar que, en este proceso de construcción, el rol de "investigadora" fue el de una actriz activa en el diálogo y en la creación de la narración. La interpretación y narración del fenómeno se construyó como "un encuentro activo entre sujetos subjetivándose" (Sisto, 2008, p. 124), en el cual se parte desde la reflexión hacia la reflexividad plasmada en las narrativas, pero no se busca analizarlas como datos recolectados como material empírico, sino como una teoría situada que es creada por las participantes en relación con su discurso sobre identidades de género. Por lo tanto, la importancia es más bien experiencial (Biglia, 2005).

Además, las producciones narrativas permitieron develar elementos teórico-conceptuales que no estaban propuestos en la investigación, y que surgieron en el tercer momento ${ }^{2}$-"la agencia"-, encontrando en el siguiente apartado los principales resultados del cuerpo narrativo que contiene las conexiones y tensiones halladas en el cruce de las narrativas de cada una de las mujeres que participaron en la elaboración de este relato.

Las producciones narrativas según Balsh y Montenegro (2003), se construyen a partir de tres actos: 1 . reflexión; 2. textualización y 3. agenciamiento. 
Con relación a las participantes, en el acceso y conformación de la muestra fueron claves los criterios de accesibilidad y homogeneidad (Valles, 1999). El primer criterio dice relación con los recursos disponibles, esto es, con el contacto realizado en el Centro de la Mujer de Talcahuano, desde el cual nace la Agrupación "Mujeres Creando Sueños", a través de la Dirección de Desarrollo Comunitario (DIDECO) de la Municipalidad de Talcahuano ${ }^{3}$. El criterio de homogeneidad de la muestra buscado se refería a la elección de mujeres a partir de un criterio base: que trabajaran por lo menos desde hace seis meses en su "intervención psicosocial" en el taller de autoayuda.

Se accedió a las mujeres y al campo de estudio por medio de procedimientos institucionales y formales ${ }^{4}$, llegando a conocer y trabajar con mujeres altamente involucradas en su intervención en violencia sexo-amorosa. El número de participantes en la construcción de la narrativa fue de nueve mujeres: ocho informantes más la investigadora.

La estrategia de construcción de narrativas se realizó en diez momentos: en los que los primeros seis encuentros consistieron en etapas de creación y levantamiento de relatos, y los cuatro siguientes en la construcción de la narrativa final.

Para el análisis de la narrativa, el procedimiento analítico utilizado fue el Método de Comparación Constante (desde ahora MCC) de análisis cualitativo. Este análisis dice relación con el enfoque de la Teoría Empíricamente Fundamentada, propuesta por Glaser y Strauss (1967), en el cual los autores establecen un proce-

Programa de violencia doméstica que tiene por objetivo la búsqueda de la reducción de la violencia contra la mujer, especialmente aquella que se produce en el contexto de relaciones de pareja. Implementa un modelo psicosocial que realiza prevención mediante trabajo comunitario y que atiende a mujeres mayores de 18 años, víctimas de violencia, derivadas por profesionales de otros servicios públicos (ejemplo CESFAM) o por demanda espontánea en Carabineros.

4 Para ello se realizaron entrevistas a la psicóloga y a la abogada del Centro de la Mujer, a partir de las cuales se pudo determinar, entre otros factores: tiempo de intervención, etapa de intervención. 
dimiento que opta por la construcción de categorías conceptuales, tanto descriptivas como interpretativas, que permiten conocer en mayor profundidad un objeto de estudio. Es importante mencionar que, para los fines de esta investigación, el análisis cualitativo realizado solo se relaciona con el procedimiento operativo y analítico de la Teoría empíricamente fundamentada, esto es, el MCC.

El MCC se distingue principalmente por generar categorías conceptuales, con propiedades (aspectos significativos de las categorías) y supuestos (o relaciones entre ellas). Las propiedades no son solo causas, sino que pueden ser "condiciones, consecuencias, dimensiones, tipos, procesos, etc." (Glauser \& Strauss, 1967, p. 104, citado en Valles, 1999, p. 347). Este método se caracteriza además por la generación de teoría en última instancia mediante un método comparativo de largo alcance, por la posibilidad de ser aplicado con cualquier tipo de información cualitativa en un mismo estudio y por la necesaria saturación de la información para delimitar categorías y propiedades (Valles, 1999), la que en esta investigación se ha construido mediante una producción narrativa.

Cabe mencionar que, considerando los fines de esta investigación, se ha optado por utilizar el MCC debido a las propiedades, flexibilidad y exhaustividad del modelo. Sin embargo, no se tiene como fin la generación de una teoría de gran alcance -fin último de la Teoría Fundamentada-, sino que se opta por una construcción de categorías conceptuales, tanto descriptivas como interpretativas, y su integración, ya que esto permite conocer en mayor profundidad el objeto de estudio propuesto, concibiendo un modelo comprensivo general de la construcción de identidad de género en la Agrupación de Mujeres.

\section{La narrativa de las mujeres en torno a su identidad}

Para las mujeres, la categoría "género" se comprende y significa a partir de dos aspectos. El primero de ellos dice relación con aspectos "rígidos o condicionantes del género", y el segundo con características y/o elementos flexibles del mismo. Dicho lo ante- 
rior, lo primero que se debe mencionar es que el concepto "género" -como tal- es una categoría que semánticamente es conocida por cada una de las mujeres que participaron en la construcción de narrativas de esta investigación; se presume que se debe a que, en su totalidad, las mujeres de la Agrupación pertenecieron a los procesos de intervención psicosocial desarrollados por el Programa Centro de la Mujer de la comuna de Talcahuano.

Dentro de los elementos "estables" del género, las mujeres hacen mención como primeros elementos al cuerpo, la binarización y la diferenciación sexual. Desde este relato, podríamos decir que ellas configuran el género desde una perspectiva tradicional, describiéndolo como aquellas características sociales y culturales asignadas a mujeres y hombres según su condición biológica sexual, respecto de las cuales los estereotipos de género asignados y construidos en el marco de una sociedad patriarcal configuran lo que las mujeres debemos ser, posicionándonos además en una estructura de relaciones de poder que se encuentra subordinada bajo modelos patriarcales y heteronormativos.

Respecto del segundo elemento desde el cual se construye el género, si bien se entiende que este es construido socialmente, tiene ápices y aristas que pueden subvertir su construcción de género y con ello su identidad. Uno de los principales factores que incide en esta construcción se relaciona con las posibilidades de transformación vinculadas con la crianza o socialización en materia de género. En este aspecto, las mujeres mencionan que, al ser las mujeres quienes tenemos en mayor grado la responsabilidad de la crianza y la educación de los hijos, se nos abre la oportunidad de poder construir identidades de género que se alejen del modelo patriarcal. Uno de los principales elementos que se menciona, además, como elemento desestabilizador del género, ha sido la incorporación de la mujer al mundo del trabajo ${ }^{5}$.

Si bien ninguna de las mujeres informantes era trabajadora asalariada al momento de realizar la recolección de datos, ellas reportaban que veían en el trabajo 
En síntesis, el género se entiende como un elemento de identificación individual y social que se compone de elementos rígidos, asociados fundamentalmente a las características anatómicas que presenta cada una de las personas, pero que podría lograr revertirse teniendo como componentes fundamentales en su trasformación a la socialización de género y a la incorporación de la mujer al mundo del trabajo.

Respecto de la construcción de identidad de género femenina, ella principalmente se articula desde lo que Sonia Montencino (1997) ha denominado el "mito mariano" y desde lo que Marcela Lagarde (1997) ha estudiado a través del "cautiverio de las mujeres". En estas perspectivas, la principal característica de la configuración de identidad de las mujeres tiene que ver con la cualidad de ser madre.

Así, el patrón sociocultural de maternidad se presenta como la realización a la que debiese aspirar cada una de las mujeres. Si bien se entiende que no todas quisieran cumplir con ello, se lamentan de no lograrlo, ya que el hecho de dar a luz se concibe como el imperativo femenino. Sin embargo, este se asume no sin culpas, ya que asimismo se menciona que su realización conlleva postergaciones, desvalorizaciones y una forma de vida.

una oportunidad de quiebre respecto de la configuración de las relaciones de género. Este último punto está conduciendo actualmente mis investigaciones. 


\section{Mapa general de codificación axial y selectiva $\mathrm{N}^{\circ} 1$}

Elementos que articulan la construcción de narrativa en torno a la identidad de género

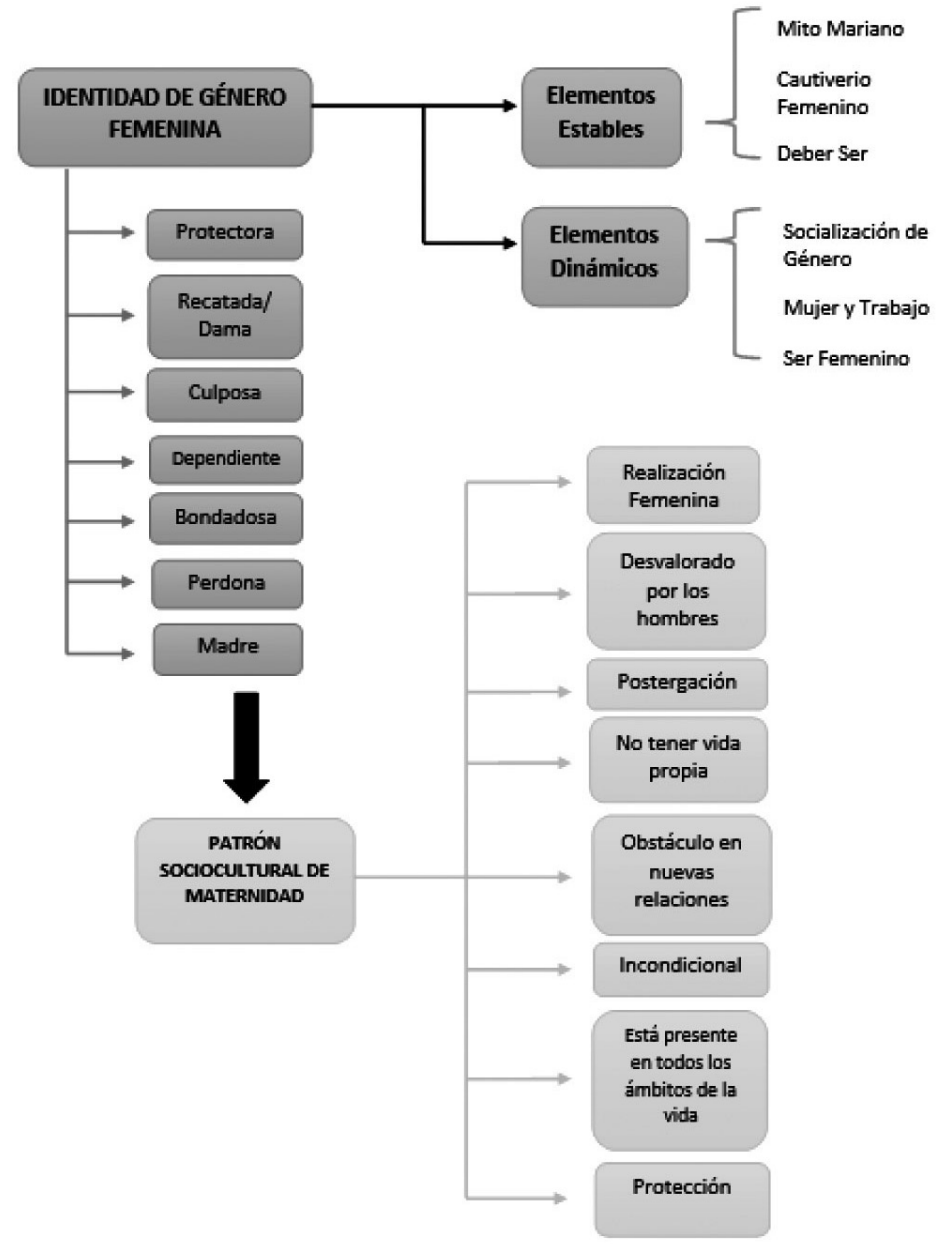

En cuanto al tema específico de la violencia sexo-amorosa, en la narrativa de las mujeres aparecen relatos asociados no solo a la violencia sexo-amorosa, sino que, además, a la de carácter institucional y sociocultural. La primera, la sexo-amorosa, se relaciona con la generación de relaciones sociales codependientes, en la cuales la violencia, en cualquiera de sus formas, se justifica a partir del amor. Respecto de la violencia institucional, las mujeres narran haberla sufrido a lo largo de todo el proceso de denuncia 
y recuperación. En primera instancia -al denunciar-, se sienten agredidas por carabineros, al ser cuestionadas en sus relatos y sindicadas como causantes de la situación de la que son víctimas; $y$, en segunda instancia, por tribunales de familia, al dilatar el proceso de juicio y además por no fiscalizar las medidas adoptadas, como por ejemplo las medidas cautelares de protección a víctimas; finalmente, también por el programa que las acogió durante todo el proceso de denuncia y recuperación -el Centro de la Mujer de Talcahuano-, que se ha visto sometido a un cambio continuo de sus profesionales, lo que no ha favorecido el proceso de recuperación de las mujeres debido a la discontinuidad de su intervención. Sin embargo, este último punto ha jugado como herramienta facilitadora de la autonomía, el empoderamiento y la creación de la Agrupación "Mujeres Creando Sueños".

Mapa general de codificación axial y selectiva $n^{\circ} 2$

Elementos que articulan la construcción de narrativa en torno a la violencia de género

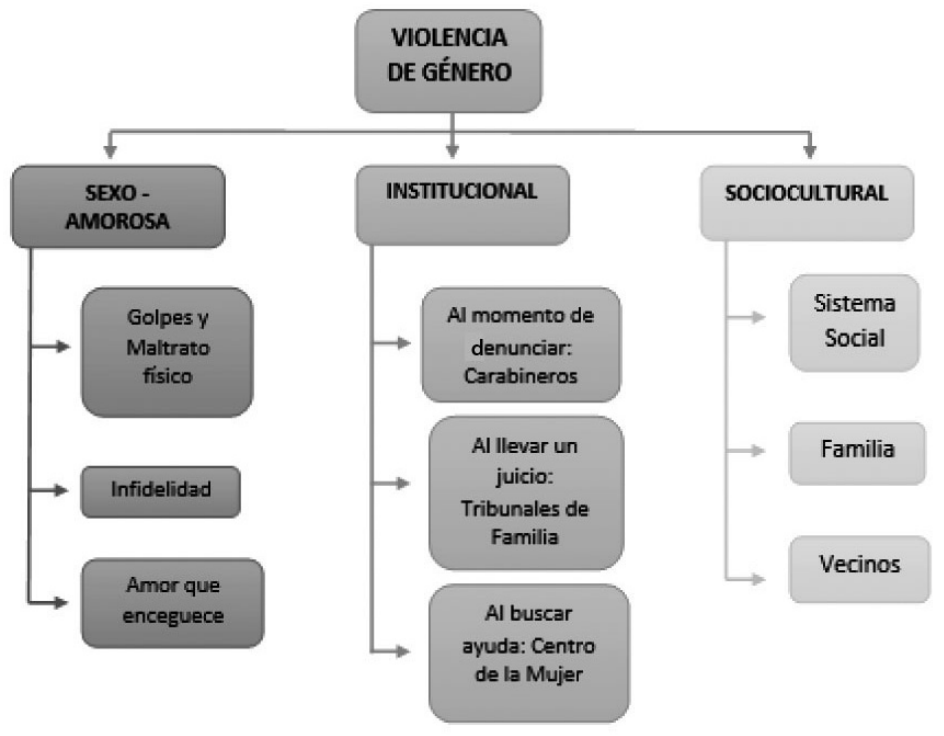

Este último punto dio vida a la última categoría, y se relaciona precisamente con el fortalecimiento que han encontrado las mujeres al compartir sus experiencias y cómo ellas han incidido en la construcción de su identidad de género. 
Mapa general de codificación axial y selectiva $n^{\circ} 3$

Elementos que articulan la identidad de la Agrupación "Mujeres Creando Sueños" de la comuna de Talcahuano

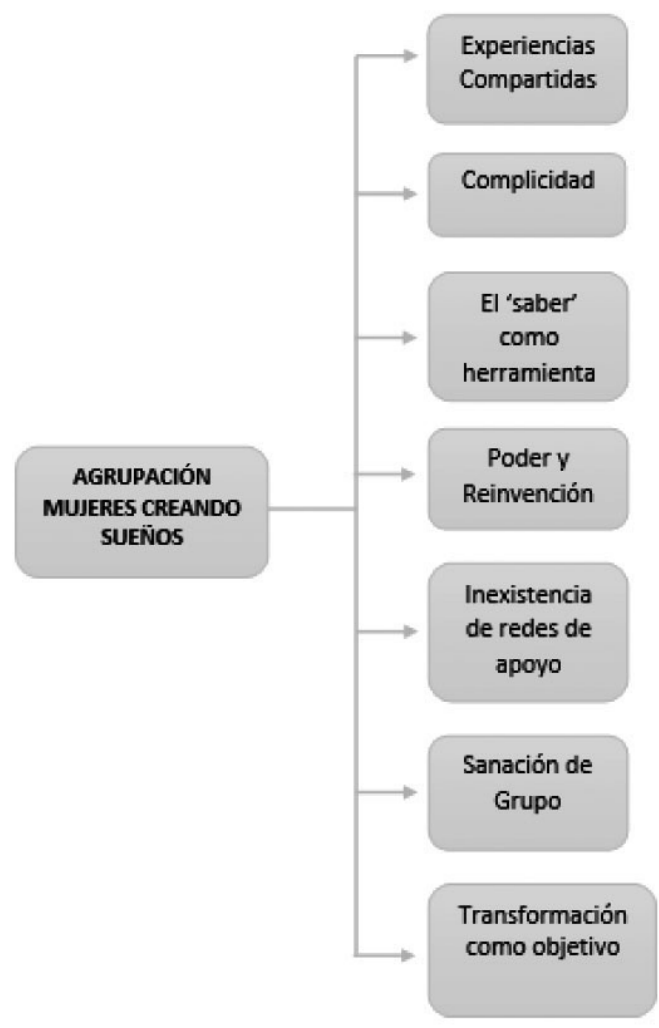

Performatividad y transformación de la identidad de género en mujeres víctimas de violencia doméstica

Los actos de habla (Austin, 1982; Butler, 2008) generan un nuevo estado de cosas, una nueva forma de autoconceptualizarse y autocomprenderse, formas que se encuentran y articulan por la práctica reiterativa y referencial mediante la cual el discurso produce los efectos que nombra, y que se hace efectiva y cada vez más potente mediante su ejercicio de repetición. 
Pero esta nominación y reiteración (Butler, 2008) también están ligadas a dispositivos de poder que, en el caso de las mujeres, se relacionan con las posibilidades de reiterar normas, patrones, modelos y prácticas instituidas y socializadas desde el modelo hétero-patriarcal. A partir de ello, entonces, la fuerza de la performatividad y de los actos del habla no radica ni en el habla ni en el lenguaje per se, sino en su capacidad de reiteración, en la persistencia, en la búsqueda de la estabilidad y en cómo ella instala formas de conocimiento y crea diversas realidades.

La construcción social de la identidad de género en las mujeres se presenta desde la tensión patriarcal, y es en la reiteración performativa que se van generando resignificaciones de las que hemos sido objeto (Íñiguez, 2001; Pujal, 2004; Butler, 2008).

En ello, podemos identificar una profunda cercanía a los patrones de género tradicionalmente asociados al mundo de lo femenino, en los que resaltan las conceptualizaciones de marianismo (Montecino, 1997) y cautiverio femenino (Lagarde, 1997), aunque estas varían en intensidad. En ellas, y para el "desarrollo de la feminidad", la maternidad es el gran eje sobre el cual se articula la vida de las mujeres, se configura el sentido de vida y es un motivo de existencia y desarrollo. Tanto en sus relatos como en su narrativa, las mujeres perciben cierta omnipotencia en su rol de madres, se plantean irremplazables, reproduciendo y ratificando, mediante diversos ejercicios de reiteración, el discurso relativo a la moral hegemónica materna -en general, respecto de su altruismo-, y la conservación y reproducción del patrón, fundamentalmente con sus hijas.

Otro elemento que se articula desde la reiteración y la estabilidad del género es que, en el caso de las mujeres de este estudio, la diferencia sexual es el factor a partir del cual se establece la diferencia entre hombres y mujeres, es decir, el género estaría determinado por el sexo, reafirmando el discurso y relato de que es la diferencia sexual la que produce una binarización de atribucio- 
nes -entre ellas de poder- a partir de la cual hombres y mujeres nos relacionamos de manera diferenciada (Badinder, 1992).

Pero, como se ha advertido, la performatividad, además de generar estabilidad mediante actos de repetición, también genera posibilidades de transformación. El lenguaje es un campo dinámico, abierto constantemente a las posibilidades de transformación y desplazamiento, "una apelación a la cita en donde la reiteración es desplazada, otorgándole a cada acto de habla su singularidad y brindándole la posibilidad de decir algo nuevo" (Morales, 2014, p. 347), siendo la comunidad de mujeres -“Mujeres Creando Sueños"-, el grupo y la posibilidad de compartir experiencias relacionadas con la violencia doméstica, quienes desencadenan este proceso de transformación.

La transformación, por tanto, también se encauza hacia aspectos vinculados con características tradicionales asociadas a la maternidad, en los que se incorporan componentes pragmáticos asociados a la necesidad del "ser" mujer, tales como la necesidad de lograr romper con ciertos patrones patriarcales en la crianza de sus hijos, así como la valorización positiva que se realiza de la incorporación de la mujer al mundo del trabajo, entendido este último como una oportunidad de independencia y un elemento que tiende hacia la transformación de las relaciones asimétricas del género, $\mathrm{y}$, con ello, al encuentro de la igualdad de derechos entre hombres y mujeres ${ }^{6}$. En este punto, es preciso destacar la contradicción identitaria que les produce este patrón, ya que si bien relatan disfrutar de su maternidad y logran definirla como "la mejor experiencia que han tenido a lo largo de sus vidas", entregan muestras explícitas de que no se sienten completas con la maternidad, en el sentido de que

Es importante mencionar que, al momento de construir estas narrativas, ninguna de las ocho mujeres participantes trabajaba, y que tampoco se visualizó como un deseo que ellas quisieran concretar en el corto/mediano plazo. Pese a ello, relataron experiencias cercanas de mujeres que trabajaban, y veían en ellas otras condiciones sobre las que se configuraba su identidad de género. 
este patrón las reduce y condiciona, y que desde esa perspectiva sienten insatisfacción porque no socializan sobre la base de virtudes, defectos personales y limitaciones, sino del sexo con el que les tocó nacer (Moore, 2004).

En lo que respecta a la construcción de relaciones sexo-amorosas, es la reiteración del relato de la complementariedad entre mujeres y hombres lo que convierte en un espejismo la generación de relaciones simétricas entre los cuerpos. Se fortalecen roles y estereotipos de género que justifican y envuelven, mediante el "poder del amor" entregado por las mujeres y la mala administración del mismo, una serie de dispositivos de control y poder con que los hombres dominan y cautivan a sus parejas y que, en conjunto con el sistema sociopatriarcal, reducen a la ejecución y reiteración de prácticas asociadas a patrones socioculturales tradicionales de género (Jonasdottir, 1993; Butler, 2008). A pesar de ello, nuevamente en tensión performativa, se encuentra la maternidad, ya que es esta -a través del amor por las y los hijos- el recurso que les permite liberarse de la violencia y no permitirse volver a recaer en una situación de similares características, y con ello reafirmar la idea de que aquello que se constituye en el discurso no es algo estable ni intransmutable, sino que es una condición que también genera oportunidades de acción (Corsi et al., 1995; Cabral y García, 2012).

Uno de los nuevos hallazgos en la construcción de las narrativas, fue la mención de la violencia institucional y sociocultural de la que son víctimas las mujeres que lo han sido también de violencia sexo-amorosa. El primer tipo de violencia se detecta en los episodios de denuncia de la situación de maltrato; el segundo, durante y con posterioridad al periodo de violencia conyugal, al generarse aislamiento, incomprensión, cuestionamiento y hostilidad desde sus redes afectivas y familiares. En ello, lo que podemos ver, es la manifestación y fuerza del sistema social patriarcal en nuestra sociedad y cómo culpabiliza a una mujer que es víctima, y la responsabiliza frente a los malos tratos que recibe (La Morada, 2013). 
Por otro lado, la visión de comunidad en las mujeres es relevante para la construcción de su identidad de género, ya que esta visión se plantea como parte de sus experiencias y de su proceso de empoderamiento y transformación. El sentido de comunidad lo podemos relacionar con la experiencia de diversas situaciones de violencia, que las lleva a la formación y autodenominación de un "nosotras las mujeres que sufrimos violencia" frente a las "mujeres que no la sufren". En este último punto, debemos reconocer que la comunidad se caracteriza por una falta de apoyo institucional (Centro de la Mujer, Municipalidad de Talcahuano, Servicio Nacional de la Mujer -SERNAM-, Biobío) y por carecer de vínculos con otras asociaciones de mujeres. Según la narrativa de las mujeres, esto produce desilusión y desde su visión creen que podría afectar la continuidad temporal de la comunidad. Este aspecto es de vital importancia, más aún si consideramos que el apoyo social otorga un sentido de pertenencia y de integración a una estructura social amplia, a la comunidad, sus instituciones y asociaciones. De este modo, el apoyo entre organizaciones de diferentes y similares objetivos posibilitaría el proceso de transformación de identidad de género y superación de la violencia sexo-amorosa (López Cabanas y Chacón, 1997).

La comunidad se establece como un espacio de agenciamiento, un campo en el que existe posibilidad para la transformación y el cambio, una reconstrucción de los referentes propios del género, a partir de la reflexión que se genera cuando se ven esos referentes estables en otra mujer. En este sentido, adquieren importancia los saberes compartidos (Montenegro, 2004). Si bien cada una de las experiencias son diversas y únicas, desde ellas se puede generar un espacio de transformación, y ese espacio está relacionado con la construcción de una nueva identidad de género que, si bien y como hemos revisado, está fuertemente ligada al patrón de maternidad, de manera muy pausada, pero cada vez más ampliada, se flexibiliza y encuentra en el conocimiento una fuente de oportunidades de cambio, a 
través de los sentimientos de filiación, influencia, integración y conexión emocional (García, Guiliani y Wiesenfeld, 1994, citado en Montenegro, 2004; Butler, 2008).

\section{Comentarios finales}

Como hemos revisado, la construcción de narrativas de las mujeres se configura principalmente en torno al patrón de maternidad, tensionado fuertemente por características ligadas al marianismo y al cautiverio de las mujeres, definido por Lagarde (1997). La construcción de la identidad de estas mujeres se configura a partir de las prácticas y dispositivos asociados al género femenino, desde una perspectiva teórica y práctica de corte tradicional, en los que la maternidad es una condición intrínseca al sexo y se establece mediante la reiteración y la nominación del "deber ser femenino".

La flexibilidad, ruptura y posibilidad de cambio, se presentan fundamentalmente desde las prácticas performativas que se generan en el discurso de las mujeres, tras el conocimiento que van adquiriendo y compartiendo en lo que a materia de género se refiere. Se comprende que este es un elemento socialmente construido; que, por ende, hay ciertos aspectos del mismo que son modificables y que, en el caso de los relatos de las mujeres, se traducen en una flexibilización de las tareas domésticas transmitidas y socializadas en la crianza de los hombres.

La identidad de género femenina en las mujeres se construye en un doble proceso, en el que la diferenciación y la semejanza construyen realidad, pero no solo con el otro masculino, sino que también con sus pares de la comunidad de mujeres. En este sentido, la observación de la proyección del comportamiento particular de cada mujer en la experiencia de otra, permite visibilizar y cuestionar un comportamiento que en ella se encuentra naturalizado y que debe romperse, sobre todo en lo que respecta a las labores domésticas y a los estereotipos de género ligados a ellas. 
La maternidad, si bien es una condición que se disfruta y se convierte en el máximo producto de la realización femenina, se presenta como un cautiverio. Un deber ser que condiciona el ser femenino y lo reduce al campo de la entrega, la servidumbre y la postergación. Respecto del comportamiento femenino esperado, son las propias mujeres las que actúan como "policías del género", esperando y exigiendo comportamientos adecuados según su sexo; por ejemplo, en el ámbito de la sexualidad, una actitud sumisa y pasiva.

Respecto de las relaciones sexo-amorosas, mencionar que la dependencia y la inadecuada administración del amor posterga y condiciona el actuar de las mujeres y su vida en pareja, siendo los afectos y el poder que les concede la maternidad sus herramientas de lucha al interior de sus relaciones afectivas.

En cuanto a la importancia de la comunidad como elemento participante en la construcción de identidad de género, se puede decir que es fundamental, en la medida en que da sentido, articula y entrega conocimiento a cada una de las mujeres.

Se construye de manera muy pausada pero latente una nueva e intersubjetiva identidad de género, una nueva subjetivación construida desde el compartir de los diversos aprendizajes y experiencias, desde el cual cada mujer es apoyada por otra, que entiende y empatiza emocionalmente con otra hasta hace un tiempo desconocida.

Como hallazgos de objetivos no propuestos, se puede mencionar la aparición de la violencia en su corte institucional y sociocultural, (formas) que en su conjunto responden a la fuerza del patriarcado y a las escasas redes de apoyo con las que cuentan las víctimas de violencia. A partir de ello, considero que se abren puertas como futuros nichos de investigación en el ámbito de la violencia a nivel estructural. Como otro tema importante a estudiar, se instala en la relación entre maternidad y trabajo femenino.

Al entender la construcción de identidad de género femenina, en el caso de las mujeres víctimas de violencia sexo-amorosa 
de la Agrupación Mujeres Creando Sueños de Talcahuano, se establece como urgente la consideración y valoración del conocimiento y la conexión emocional compartida de experiencias dentro de las intervenciones a realizar por las políticas públicas, esto pensando que la identidad se construye de manera social, y que es de la misma forma que se pueden generar transformaciones, más aún considerando el peso y naturalización de la maternidad en el ser y deber ser de las mujeres.

\section{Referencias}

Austin, J. (1982). Cómo hacer cosas con palabras. España: Paidós.

Badinter, E. (1992). ¿Existe el instinto maternal? Historia del amor maternal (S. XVII-XX). España: Paidós.

Balash, M. y Montenegro, M. (2003). Una propuesta metodológica desde la epistemología de conocimientos situados: las producciones narrativas. Encuentros en Psicología Social 1(1), 44-48.

Biglia, B. (2005). Narrativas de mujeres en torno a relaciones de género en movimientos sociales. Tesis para optar al título de Doctora en Psicología Social. Universidad de Barcelona, España.

Blanchs, M. (2010). Identidades de género en la encrucijada de la sociedad matrilineal al umbral de la posmodernidad. En M. Quintero (coord.). Identidad y Alteridades - Mérida, Fascículo de AVEPSO, 10, 49-67.

Butler, J. (2008). Cuerpos que importan. Sobre los límites materiales y discursivos del "sexo". Segunda Edición. Argentina: Paidós.

Cabral, B. y García, C. (2012). Violencia de género: saberes, prácticas sociales y estrategias de poder. Recuperado de: http:/ /www.saber. ula.ve/db/ssaber/Edocs/pubelectronicas/revistacenipec/cenipecnum18-19/articulo1.pdf

Corsi, J. et al. (1995). Violencia masculina en la pareja. Una aproximación al diagnóstico y a los modelos de intervención. Argentina: Paidós.

Gadamer, H. (1975). Verdad y Método I. Salamanca, España: Editorial Sígueme.

Haraway, D. (1991). Conocimientos situados: la cuestión científica en el feminismo y el privilegio de la perspectiva parcial. En D. Haraway (ed.). Ciencia, Cyborgs y Mujeres: La reinvención de la Naturaleza. Madrid: Ediciones Cátedra, 183-201. 
Ibáñez, T. (1994). Poder y Libertad: Estudio sobre la naturaleza, las modalidades y los mecanismos de poder. Recuperado de: http://148.201.94.3:8991/F?func=directat_ base $=$ ITE01\&doc_number $=00015311$

Ibáñez, T. (2001). Municiones para disidentes: Realidad, verdad, política. Barcelona, España: Editorial Gedisa.

Íñiguez, L. (2001). Identidad: de lo personal a lo social. Un recorrido conceptual. En E. Crespo y C. Soldevilla (eds.). La constitución social de la subjetividad. Madrid: Los Libros de la Catarata, 209-226.

Jónasdóttir, A. (1993). El poder del amor. ¿Le importa el sexo a la democracia? Valencia, España: Editorial Cátedra.

La Morada (2013). Derecho de las humanas: concurso de ensayos. Santiago de Chile: Corporación de Desarrollo de la Mujer.

Lagarde, M. (1997). Los cautiverios de las mujeres: madresposas, monjas, putas, presas y locas. México D.F.: UNAM, colección Posgrado.

López-Cabanas, M. y Chacón, F. (1997). Intervención psicosocial y servicios sociales. Un enfoque participativo. Madrid: Editorial Síntesis.

Marinas, J. (2007). La escucha en la historia oral. Palabra Dada. Madrid, España: Editorial Síntesis.

Martínez-Guzmán, A. y Montenegro, M. (2009). Narrativas en torno al trastorno de identidad sexual. De la multiplicidad transgénero a la producción de transconocimientos. Revista de Ciencias Sociales, 4, junio, 1-44.

Montecino, S. (1997). Palabra dicha. Ensayos sobre identidades, género y mestizaje. Disponible en red: http://rehue.csociales.uchile. $\mathrm{cl} /$ paginas_academicos/montecino/publicaciones.html

Montenegro, M. (2001). La intervención social: intervenciones dirigidas. Tesis de Doctorado: Conocimientos, Agentes y Articulaciones, Una Mirada Situada a la Intervención Social. Barcelona: Universidad Autónoma de Barcelona.

Montenegro, M. (2004). Comunidad y bienestar social. En J. Herrero; L. Cantera y M. Montenegro. Introducción a la psicología comunitaria. España: Ed. UOC.

Montenegro, M. (2004) (b) La lucha por los significados, conceptos y teorías sobre problemas sociales. En Psicología social de los problemas sociales. Santiago de Chile: Universidad de Chile. 
Moore, H. (2004). Antropología y feminismos. Valencia, España: Ediciones Cátedra.

Morales, M. (2014). Discurso, perfomatividad y emergencia del sujeto: Un abordaje desde el post-estructuralismo. Revista Athenea Digital, 14 (1), 333 -354.

Pujal, M. (2003). La identidad (el self). En T. Ibáñez et al. Introducción a la psicología social. Barcelona: Editorial UOC.

Sisto, V. (2008). La investigación como una aventura de producción dialógica: La relación con el otro y los criterios de validación en la metodología cualitativa contemporánea. Revista Psicoperspectivas, VII, 114-136.

Straus A. y Corbin, J. (2002). Bases de la Investigación cualitativa. Medellín: Editorial de la Universidad de Antioquia.

Valles, M. (1999). Técnicas cualitativas de investigación social: reflexión metodológica y práctica profesional, Madrid: Editorial Síntesis. 